\title{
New Kinematics for the Central Spheroid in Polar Disk Galaxy NGC4650A
}

\author{
E. Iodice ${ }^{1}$, M. Arnaboldi ${ }^{2}$, R. Saglia ${ }^{3}$, L. Sparke ${ }^{4}$, \\ and O. Gerhard ${ }^{3}$, et al. \\ ${ }^{1}$ INAF-Astr. Obs. of Capodimonte, Napoli, Italy - email: iodice@na.astro.it; \\ ${ }^{2}$ ESO - Garching, Germany; ${ }^{3} \mathrm{MPE}$, Garching, Germany; ${ }^{4}$ Univ. of Wisconsin, Madison, US
}

\begin{abstract}
We have obtained high angular resolution spectra on the photometric axes of the stellar spheroid in the polar disk galaxy NGC4650A. We discuss the main implications by the new kinematics on the previous mass models for NGC4650A and on current formation scenarios of Polar Ring Galaxies (PRGs).
\end{abstract}

Keywords. galaxies: formation - galaxies: kinematics and dynamics

\section{Results and discussion}

NGC4650A is the prototype for PRGs. Its luminous components, inner spheroid and polar structure, have been studied with optical and NIR photometry, optical spectroscopy and in the radio. The central spheroid is an exponential thick disk, characterized by a very bright nucleus; the polar structure has been shown to be a disk, rather than a ring. Up to date, PRGs represent a unique laboratory to study many of the processes involved in galaxy interactions and merging and to constraint the 3D dark halo shape. To this aims, we have obtained high resolution spectra (with FORS2 on the ESO VLT) of the Calcium triplet absorption lines on the photometric axes of the stellar spheroid in NGC4650A: the new results (also mentioned below) were published by Iodice et al. (2006). Along the major axis, the observed rotation and velocity dispersion measurements show the presence of a kinematically decoupled nucleus, and a flat velocity dispersion profile. The minor axis kinematics is determined for the first time: along this direction some rotation is measured, and the velocity dispersion is nearly constant at all radii.

The new high resolution kinematic data suggest that the stellar component in NGC4650A resembles a nearly-exponential oblate spheroid supported by rotation. The main implications of these results on the previous mass models for NGC4650A concern the law adopted to fit the velocity dispersion profile: the measured flat velocity dispersion profile along the spheroid major axis shows that both the linear decreasing fit and the exponential empirical law adopted in the previous mass models do not reproduce the observed trend with radius and previous conclusions on the halo flattening are no longer valid.

Moreover, the new kinematic data set constraints on the current formation scenarios for PRG: the observed kinematics along the major axis does not reconcile with the predictions by the merging scenario, which suggests that the spheroid in a PRG with a massive polar disk, as observed in NGC4650A, is an elliptical-like not rotationally supported system. Differently, the new kinematics supports the slow accretion mechanism: both through tidal accretion and cold gas accretion along a filament, a polar ring and/or disk may form both around a disk or an elliptical galaxy.

\section{Reference}

Iodice, E., Arnaboldi, M., Saglia, R., Sparke, L., Gerhard, O., et al. 2006, ApJ 643, 200. 